\section{(6) OPEN ACCESS}

\title{
High prevalence of high-risk HPV genotypes other than 16 and 18 in cervical cancers of Curaçao: implications for choice of prophylactic HPV vaccine
}

\author{
Desiree J Hooi, ${ }^{1,2}$ Birgit I Lissenberg-Witte, ${ }^{3}$ Maurits N C de Koning, ${ }^{4}$ \\ Herbert M Pinedo, ${ }^{1}$ Gemma G Kenter, ${ }^{5}$ Chris JLM Meijer, ${ }^{2}$ Wim G Quint ${ }^{4}$
}

\begin{abstract}
- Additional material is published online only. To view please visit the journal online (http://dx.doi.org/10.1136/ sextrans-2017-053109).

${ }^{1}$ Fundashon Prevenshon, Willemstad, Curaçao ${ }^{2}$ Department of Pathology, VU University Medical Centre, Amsterdam, the Netherlands ${ }^{3}$ Department of Epidemiology and Biostatistics, VU University Medical Centre, Amsterdam, the Netherlands

${ }^{4}$ DDL Diagnostic Laboratory, Rijswijk, the Netherlands ${ }^{5}$ Department of Gynaecology and Oncology, VU University Medical Centre, Amsterdam, the Netherlands
\end{abstract}

\section{Correspondence to} Dr Chris JLM Meijer, VUmc Cancer Centre Amsterdam, De Boelelaan, Amsterdam 1118 The Netherlands; cjlm.meijer@ vumc.nl

Received 18 January 2017 Revised 21 July 2017 Accepted 8 August 2017 Published Online First 11 October 2017
Check for updates

To cite: Hooi DJ, LissenbergWitte $\mathrm{BI}$, de Koning MNC et al. Sex Transm Infect 2018:94:262-266.

\begin{abstract}
Background Curaçao is a Dutch-Caribbean Island located in a high-risk area for cervical cancer. Prior to introduction of a prophylactic human papillomavirus (HPV) vaccine, knowledge of the prevalence of high-risk HPV vaccine genotypes (HPV16, 18, 31, 33, 45, 52 and 58 ) in cervical (pre)cancer is required.

Objective To investigate the prevalence of HPV genotypes in invasive cervical cancers (ICC) and cervical intraepithelial neoplasia (CIN) grade 1, 2 and 3 in Curaçao.

Methods Paraffin-embedded blocks of 104 cervical cancers (89 squamous, 15 adenocarcinoma), 41 CIN3, 39 CIN2 and 40 CIN1 lesions were analysed for the presence of HPV. Sections were stained by H\&E for histopathological evaluation, and DNA was extracted using proteinase K. HPV genotypes were detected using Short PCR Fragment (SPF10) PCR DNA enzyme immunoassay and a Line Probe Assay (LiPA25) .
\end{abstract}

Results HPV was found in 92 (88.5\%) ICC; 87 (94.6\%) had a single HPV infection and $86(93.5 \%)$ were highrisk human papillomavirus (hrHPV)-type positive. The three most common HPV types in ICC were 16 (38.5\%), $18(13.5 \%)$ and $45(6.7 \%)$, covering $58.7 \%$. HrHPV vaccine genotypes 16, 18, 31, 35, 45, 52 and 58 were responsible for $73.1 \%$ of ICC. For precancerous lesions, the HPV attribution was $85.4 \%$ for CIN3, $66.7 \%$ for CIN2\% and $42.5 \%$ for CIN1.

Conclusions Our study, the largest in the Caribbean region in (pre)cancer, shows that the prevalence of HPVtype 16 and 18 in cervical cancer is lower compared with the world population but no differences in prevalence of these two HPV types are seen in precancerous lesions. When considering HPV vaccination in Curaçao, the relatively high contribution of non-HPV 16/18 genotypes in ICC should be taken into account.

\section{INTRODUCTION}

Curaçao is a Dutch-Caribbean island with estimated 155000 inhabitants last registered in 2014 . $^{1}$ It is demographically located in an area with a high incidence and mortality of cervical cancer ${ }^{2-5}$ known to be caused by high-risk HPV. ${ }^{67}$ According to The Cancer Registry of Curaçao, the incidence of cervical cancer over 2004-2008 is 13.4 per 100000 women (CMD Coronel, personal communication, 2015).
The lack of an organised cervical screening and prophylactic HPV vaccination programme largely explains the high incidence of cervical cancer. ${ }^{8-10}$

Presently, three HPV prophylactic vaccines are available. A bivalent HPV vaccine against HPV16 and 18; a quadrivalent vaccine directed against HPV16 and 18 with an additional coverage to low-risk HPV 6 and 11; and recently a nonavalent vaccine directed against high-risk Human papillomavirus (hrHPV)16, 18, 31, 33, 45, 52 and 58 and 1rHPV6 and 11. The bivalent and quadrivalent vaccines have shown partial to full cross-protection against certain non-vaccine HPV types. ${ }^{11} 12$

According to the report about the situational analysis of cervical cancer prevention and control in the Caribbean, only three Caribbean islands have a HPV vaccination programme up and running. ${ }^{10}$ In 2007, the first HPV vaccine was introduced on Curaçao. Both the bivalent and quadrivalent HPV vaccines are available and recently in 2017, the nonavalent HPV vaccine was introduced. These vaccines are not part of a National Vaccination Programme and are only available at their own expense. Family practitioners and paediatricians can prescribe the vaccines on patient's request. At present, there are no standard HPV vaccination protocol or a national vaccination registry functioning. Less than a hundred vaccines were sold since the introduction in 2007 , until resulting in a very low vaccine coverage (personal communication by S. Elhage, M. Ruijs and J. Boujon of the local vaccine agencies).

In July 2016, the island started with an HPV-based national cervical cancer screening programme.

Before introducing a prophylactic vaccine to women on Curaçao, it is important to know the prevalence of HPV genotypes in cervical precancerous lesions and cancer. This can help in the decision by policymakers which prophylactic HPV vaccine should be used and what the potential impact of the new vaccine might be. Here we describe the prevalence of HPV genotypes in paraffin-embedded tissues of cervical cancers and cervical precancerous lesions (cervical intraepithelial neoplasia grades 1,2 and 3 (CIN1, CIN2, CIN3)) derived from the only pathology laboratory of Curaçao. 
Table 1 Type of tissue sample of cervical cancer and CIN lesion used for HPV detection

\begin{tabular}{lllll}
\hline & Cervical ca. & CIN3 & CIN2 & CIN1 \\
\hline Biopsies & 75 & 30 & 27 & 25 \\
\hline $\begin{array}{l}\text { Large loop excision of the } \\
\text { transformation zone (LLETZ) }\end{array}$ & 1 & 5 & 6 & 4 \\
Intracervical curettages & 6 & 4 & 3 & 2 \\
Cervix conisations & 14 & 1 & 2 & 8 \\
Hysterectomies & 8 & 0 & 1 & 2 \\
Total & 104 & 40 & 39 & 41 \\
\hline
\end{tabular}

CIN, cervical intraepithelial neoplasia.

\section{MATERIAL AND METHODS Material}

A retrospective study was designed and co-ordinated by Fundashon Prevenshon (FP), Curaçao, in co-operation with DDL Diagnostic Laboratory, Rijswijk, the Netherlands; the department of pathology, VU University Medical Centre (VUmc) Amsterdam, the Netherlands and the Analytic Diagnostic Centre (ADC), Curaçao, to determine the prevalence of HPV DNA genotypes in formalin-fixed paraffin-embedded (FFPE) tissues of women with invasive cervical cancer (ICC).

All ICC and CIN cases diagnosed in woman from Curaçao were selected in the PALGA system, which is the nationwide network and registry of histopathology and cytopathology in the Netherlands and Curaçao. This system includes both private and government insured patients. Only available blocks of good quality, containing enough tissue, were selected from 2012 backwards, anonymised, relabelled and analysed at DDL diagnostic laboratory. In total, FFPE blocks of 104 cervical cancers, 40 CIN1, 39 CIN2 and 41 CIN3 were collected from the ADC's department of Pathology, Curaçao. The type of tissue from which the lesion samples are obtained is given in table 1 .

Eight sections were cut from each block according to the sandwich method for histopathology and DNA extraction for HPV testing. ${ }^{7}$

In brief, the first and last sections were $4 \mu \mathrm{m}$ and stained with $H \& E$ for histopathological evaluation of the lesion. In between the two H\&E-stained sections, six sections of $8 \mu \mathrm{m}$ were cut. The first three were combined in a single tube and used for HPV genotyping, the last three were stored in another tube for back up.

After each 10 blocks that were cut, an empty paraffin block was cut as a control for contamination during the sectioning process. Sections from the control block were tested in parallel with the samples and remained negative. For each sample, we also obtained age of the woman at diagnosis, date of sample collection and histological diagnosis.

\section{DNA isolation and HPV testing}

Tissue sections were incubated overnight for DNA extraction with $250 \mu \mathrm{L}$ of a $0.1 \%$ proteinase $\mathrm{K}$ solution at $70^{\circ} \mathrm{C}$. The standard proteinase volume of $250 \mu \mathrm{L}$ was reduced to $100 \mu \mathrm{L}$ for sections containing small tissue samples as observed by visual inspection. After incubation, proteinase $\mathrm{K}$ was inactivated at $95^{\circ} \mathrm{C}$ for $10 \mathrm{~min}$. HPV DNA was amplified by the SPF10 PCR in a total volume of $50 \mu \mathrm{L}$ using $10 \mu \mathrm{L}$ of 10 times diluted DNA. The SPF10 DNA enzyme immunoassay (DEIA) (Labo Bio-medical Products, Rijswijk, The Netherlands) was used for general detection of over 69 known HPV types by hybridisation to a mixture of HPV-specific probes in a 96-well format. $^{1314}$

Amplimers from positive samples by DEIA were genotyped in the SPF10LiPA25 reverse hybridisation assay for identification of $25 \mathrm{HPV}$ genotypes ${ }^{15}$ (version1; Labo Bio-medical Products, based on licensed Innogenetics technology).

DNA from all samples was also tested for the presence of amplifiable human genomic DNA and PCR inhibition by a duplex real-time PCR targeting the human RNaseP gene and a plasmid spiked into the PCR mix. ${ }^{16}$ In case the Ct value of the plasmid spike-in was elevated, indicating PCR inhibition, the already 10 times diluted sample was diluted 10 more times and re-tested for HPV and PCR inhibition. If the 10 times diluted sample did not show PCR inhibition and the HPV result was negative, the undiluted sample was re-tested for HPV and PCR inhibition.

\section{Statistical analyses}

HPV type attribution per group was assessed in different ways to deal with multiple infections (see online supplementary table S1). First, we considered only single HPV infections. That is, women with multiple HPV infections were not included in the calculation, underestimating the genotype attributable fraction. Second, we considered each infection as a causal infection, that is, for a woman infected with HPV16 and HPV31, both 16 and 31 were counted as separate attribution, overestimating the genotype attributable fraction. ${ }^{17}$ Finally, we used hierarchical and proportional attribution to account for multiple HPV infections. ${ }^{18}$ In short, in a woman with multiple infections, either the HPV infection with the highest prevalence within the population (hierarchical method) or each HPV infection proportional to the total prevalence within the population (proportional method) is assumed to have attributed to the lesion.

Ethical approval of the study was obtained from the institutional review board (IRB) of the medical ethics committee of FP, Curaçao (IRB board's approval number 0001/14).

Table 2 The prevalence of HPV, single HPV and multiple HPV infections and hrHPV in cervical cancer and CIN lesions

\begin{tabular}{|c|c|c|c|c|c|c|c|c|c|c|c|c|c|}
\hline & $\mathrm{N}$ & $\begin{array}{l}\text { Age } \\
\text { (mean) }\end{array}$ & $\begin{array}{l}\text { Age } \\
\text { (range) }\end{array}$ & $\begin{array}{l}\text { HPV } \\
\text { positive }\end{array}$ & $\%$ & $\begin{array}{l}\text { HPV } \\
\text { negative }\end{array}$ & $\%$ & $\begin{array}{l}\text { HPV } \\
\text { single }\end{array}$ & $\%$ & $\begin{array}{l}\text { HPV } \\
\text { multiple }\end{array}$ & $\%$ & $\begin{array}{l}\text { hrHPV* } \\
\text { pos. }\end{array}$ & $\%$ \\
\hline CIN1 & 40 & 32.7 & $(20-51)$ & 31 & 77.5 & 9 & 22.5 & 21 & 67.7 & 10 & 32.3 & 24 & 77.4 \\
\hline CIN2 & 39 & 34.4 & $(19-72)$ & 35 & 89.7 & 4 & 10.3 & 27 & 77.1 & 8 & 22.9 & 34 & 97.1 \\
\hline CIN3 & 41 & 38.6 & $(25-74)$ & 39 & 95.1 & 2 & 4.9 & 31 & 79.5 & 8 & 20.5 & 39 & 100.0 \\
\hline Cancer (ICC) & 104 & 52.6 & $(27-86)$ & 92 & 88.5 & 3 & 20.0 & 87 & 94.6 & 5 & 5.4 & 86 & 93.5 \\
\hline $\mathrm{SCC}+\mathrm{ASC}$ & 89 & 52.2 & $(27-86)$ & 80 & 89.9 & 9 & 10.1 & 75 & 93.8 & 5 & 6.3 & 74 & 92.5 \\
\hline $\mathrm{ADC}$ & 15 & 51.9 & $(32-74)$ & 12 & 80.0 & 12 & 11.5 & 12 & 100.0 & 0 & 0 & 12 & 100.0 \\
\hline
\end{tabular}

*Percentage of hrHPV is calculated from the number of HPV-positive cases.

ADC, adenocarcinomae; ASC, adenosquamous carcinoma; CIN, cervical intraepithelial neoplasia; ICC, invasive cervical cancer; SCC, squamous cell carcinoma. 
Table 3 HPV-type distribution in HPV-positive cervical cancer, subdivided into SCC/ASC, ADC and CIN 3, 2, 1 lesions.

\begin{tabular}{|c|c|c|c|c|c|c|c|c|c|c|c|c|c|c|}
\hline \multirow[b]{3}{*}{ Type } & \multicolumn{14}{|c|}{ Hierarchical attribution* } \\
\hline & \multicolumn{2}{|c|}{ Cancer } & \multicolumn{2}{|c|}{ SCCIASC } & \multicolumn{2}{|c|}{ ADC } & \multicolumn{2}{|c|}{ CIN3 } & \multicolumn{2}{|c|}{ CIN2 } & \multicolumn{2}{|c|}{ CIN1 } & \multicolumn{2}{|c|}{ Total } \\
\hline & $n$ & $\%$ & $n$ & $\%$ & $n$ & $\%$ & $n$ & $\%$ & $n$ & $\%$ & $n$ & $\%$ & $n$ & $\%$ \\
\hline \multicolumn{15}{|l|}{ hrHPV } \\
\hline 16 & 40 & 38.5 & 36 & 40.4 & 4 & 26.7 & 19 & 46.3 & 12 & 30.8 & 6 & 15.0 & 77 & 34.4 \\
\hline 18 & 14 & 13.5 & 8 & 9.0 & 6 & 40.0 & 3 & 7.3 & 4 & 10.3 & 1 & 2.5 & 22 & 9.8 \\
\hline 31 & 4 & 3.8 & 4 & 4.5 & & & 5 & 12.2 & 2 & 5.1 & 5 & 12.5 & 16 & 7.1 \\
\hline 33 & 3 & 2.9 & 3 & 3.4 & & & 1 & 2.4 & 2 & 5.1 & 1 & 2.5 & 7 & 3.1 \\
\hline 35 & 3 & 2.9 & 3 & 3.4 & & & & & 4 & 10.3 & & & 7 & 3.1 \\
\hline 39 & 1 & 1.0 & 1 & 1.1 & & & & & 1 & 2.6 & & & 2 & 0.9 \\
\hline 45 & 7 & 6.7 & 5 & 5.6 & 2 & 13.3 & 2 & 4.9 & 1 & 2.6 & 2 & 5.0 & 12 & 5.4 \\
\hline 51 & 3 & 2.9 & 3 & 3.4 & & & 2 & 4.9 & 4 & 10.3 & 3 & 7.5 & 12 & 5.4 \\
\hline 52 & 4 & 3.8 & 4 & 4.5 & & & 3 & 7.3 & 2 & 5.1 & 1 & 2.5 & 10 & 4.5 \\
\hline 56 & & & & & & & & & & & 1 & 2.5 & 1 & 0.4 \\
\hline 58 & 4 & 3.8 & 4 & 4.5 & & & 3 & 7.3 & 1 & 2.6 & 2 & 5.0 & 10 & 4.5 \\
\hline 59 & 1 & 1.0 & 1 & 1.1 & & & & & & & 1 & 2.5 & 2 & 0.9 \\
\hline 66 & & & & & & & 1 & 2.4 & 1 & 2.6 & & & 2 & 0.9 \\
\hline 68 & 2 & 1.9 & 2 & 2.2 & & & & & & & 1 & 2.5 & 3 & 1.3 \\
\hline \multicolumn{15}{|l|}{ IrHPV } \\
\hline 6 & & & & & & & & & 1 & 2.6 & 2 & 5.0 & 3 & 1.3 \\
\hline \multicolumn{15}{|l|}{44} \\
\hline 53 & & & & & & & & & & & 1 & 2.5 & 1 & 0.4 \\
\hline \multicolumn{15}{|l|}{54} \\
\hline 61 & & & & & & & & & & & 1 & 2.5 & 1 & 0.4 \\
\hline 67 & 1 & 1.0 & 1 & 1.1 & & & & & & & & & 1 & 0.4 \\
\hline 70 & & & & & & & & & & & 2 & 5.0 & 2 & 0.9 \\
\hline \multicolumn{15}{|l|}{71} \\
\hline \multicolumn{15}{|l|}{74} \\
\hline 82 & 1 & 1.0 & 1 & 1.1 & & & & & & & & & 1 & 0.4 \\
\hline \multicolumn{15}{|l|}{91} \\
\hline X & 4 & 3.8 & 4 & 4.5 & & & & & & & 1 & 2.5 & 5 & 2.2 \\
\hline Total & 92 & 88.5 & 80 & 89.9 & 12 & 80.0 & 39 & 95.1 & 35 & 89.7 & 31 & 77.5 & 197 & 87.9 \\
\hline Negative & 12 & 11.5 & 9 & 10.1 & 3 & $20 . \%$ & 2 & 4.9 & 4 & 10.3 & 9 & 22.5 & 27 & 12.1 \\
\hline
\end{tabular}

ADC, adenocarcinomae; ASC, adenosquamous carcinoma; CIN, cervical intraepithelial neoplasia; SCC, squamous cell carcinoma.

\section{RESULTS}

Table 2 presents the mean age at which cervical cancer and CIN lesions were diagnosed, and the prevalence of HPV in these lesions.

Since the results of hierarchical attribution analysis were similar to the proportional attribution analysis, we only present here the hierarchical distribution. The prevalence of HPV genotypes in multiple infections in cervical cancer and CIN lesions is shown in online supplementary table S1.

\section{Carcinomas}

Most of the histotypes were squamous cell carcinoma (SCC $\mathrm{n}=85(81.7 \%))$, followed by adenocarcinoma ((ADC $\mathrm{n}=15$ $(14.4 \%))$ and adenosquamous carcinoma ((ASC $\mathrm{n}=4$ (3.8\%)) (table 2).

Because of the small number of ASC, for statistical analysis we added the four ASC cases to the SCC.

The large majority $(88.5 \%)$ of cervical carcinoma were HPV positive of which only $5.4 \%$ had a multiple infection. The majority of positive ICC cases $(93.5 \%)$ contained one hrHPV type (table 2). The most prevalent HPV types in ICC were 16 (38.5\%), 18 (13.5\%) and $45(6.7 \%)$, covering $58.7 \%$ of the carcinomas (table 3 ). Four out of the five multiple hrHPV infections in cervical cancer had HPV16 in combination with another highrisk type (see online supplementary table S2).

Eighty per cent of the ADC $(n=15)$ were HPV positive.

Only single HPV-type infections were detected, and all samples were high-risk HPV types. HPV18 was the most prevalent HPV type (40.0\%) followed by HPV16 (26.7\%) and HPV45 (13.3\%) (table 3).

In total, 12 of the ICC samples were HPV negative: nine SCC and three ADC.

\section{CIN lesions}

The percentages of HPV prevalence increased with the grade of CIN lesion namely CIN1 77.5\%, CIN2 89.7\%, CIN3 95.1\% (table 2). The percentages of multiple HPV infections decreased from CIN1 (32.3\%) to CIN3 (20.5\%)and were lowest in cervical carcinomas $(5.4 \%)$.

HPV16 was the most prevalent HPV type in all degrees of $\mathrm{CIN}$ and its prevalence increased substantially with greater severity of CIN. The most prevalent HPV types in CIN3 samples were HPV16 (46.3\%) and HPV 31 (12.2\%). HPV types 18, 52 and 58 each covered $7.3 \%$.

In CIN 2, the most common type was HPV16 (30.8\%), while HPV18, 35 and 51 were the second most common, each covered 
10.3\%. In CIN 1, the most common HPV types were HPV16 (15.0\%), HPV31 (12.5\%) and HPV51 (7.5\%) (table 3).

\section{DISCUSSION}

This is the first study from Curaçao to show systematic data on HPV prevalence in cervical cancer and CIN lesions. Interestingly, although the prevalences of HPV16 (38.5\%) and HPV18 $(13.5 \%)$ in cervical cancer of women from Curaçao are still the most prevalent HPV genotypes, their prevalence is lower compared with the world prevalence. ${ }^{19-21}$

The prevalence of vaccine types HPV31, HPV52, HPV58 and HPV X (DEIA pos. and LiPA neg.) for each HPV type was $3.8 \%$, in total 15.2\%. In ADC $(n=15)$, HPV $18(40.0 \%)$, HPV16 (26.7\%) and HPV45 (13.3\%) were the most prevalent HPV types. Twelve cervical cancers were found to be HPV negative.

We performed several experiments to substantiate absence of HPV in cervical cancer and exclude experimental causes. Review of the slides by two pathologists did not change the diagnosis, and repeated HPV testing and genotyping with SPF10 yielded identical results. Inadequate DNA quality could be excluded by parallel testing with a duplex real-time PCR targeting the human RNaseP gene. Potential PCR inhibition could be excluded by a plasmid spiked into the PCR mix.

All 12 samples contained amplifiable human genomic DNA and stable $\mathrm{Cq}$ values of the plasmid spike showed absence of PCR inhibition. Another explanation for a negative HPV result in cervical carcinoma might be integration of HPV DNA in the L1 region because the SPF10 targets the L1 gene. We did not perform HPV detection assays targeting the E6/E7 region to exclude this possibility. ${ }^{22}$ However, published data show that $<1 \%$ of cervical cancers has integration in the L1 region. ${ }^{23} 24$

Our HPV 16/18 prevalence data in cervical cancer are in agreement with Martinique (57\% in 131 ICC) $)^{25}$ but differ from those in Trinidad (83.9\%). ${ }^{26}$

The ranking order of HPV genotypes found in cervical cancer in Curaçao matches with those from Trinidad HPV16 (66.1\%), HPV18 (17.8\%) and HPV45 (8.9\%). ${ }^{26}$ Comparison with HPV genotype studies from Jamaica was difficult due to pooling of the small sample size (9 ICC and 30 CIN3 lesions). ${ }^{27}$

Compared with HPV 16 and 18 in cervical cancer from the Caribbean region according to the Institut Català d'Oncologia (ICO) data (57.6\%), our estimated HPV16 and 18 prevalence data in cervical cancer are somewhat lower (52.0\%), but our HPV 16 and 18 prevalence data in high-grade lesions (CIN2/3; $47.6 \%)$ are higher compared with the ICO data (CIN2/3; $34.6 \%) .{ }^{19}$

However, it should be noted that most of the published HPV prevalence data from CIN lesions in the Caribbean region are obtained from studies in women with normal cytology where these lesions were discovered incidentally during the trials. In Jamaica $(n=840)$, the prevalence of HPV16 and 18 was found to be $50 \%$ in 14 high-grade squamous intraepithelial lesion (HSIL) and $21.3 \%$ in 61 atypical squamous cells of undetermined significance (ASC-US) and low-grade squamous intraepithelial lesion (LSIL). ${ }^{28}$

Data from Guadeloupe $(n=618)$ only described HPV 16 and 18 prevalence in LSIL: $9.7 \%$ in LSIL $(n=31)$ and $11.3 \%$ of ASC-US $(n=106)$. HPV 16 and 18 prevalence in HSIL were not described because of the small sample size $(n=8) .^{29}$

An explanation for the differences in HPV prevalence between the Caribbean region may be the very heterogeneous ethnical background, which consists of a mix of African and European

\section{Key messages}

- The incidence of cervical cancer in Curaçao is high (13.1) and cervical cancer prevention programmes are lacking.

- The prevalence of HPV16 and 18 in cervical cancer in this large study from Cura? ?ao is lower compared with the world population and Trinidad.

- The prevalence of HPV genotypes in CIN lesions in Cura??ao is comparable with the world population.

- In the selection of a prophylactic HPV vaccine the high proportion of non-HPV 16/18 genotypes in cervical cancer should be taken into account.

descent and different social and cultural behaviours in this population.

Our HPV genotype prevalence data argue for the use of a prophylactic HPV vaccine with broad coverage of the non-HPV $16 / 18$ genotypes, as present in the nonavalent vaccine. The use of HPV vaccines with a smaller HPV genotype coverage such as the bivalent vaccine can also be considered when longitudinal data show that the cross-protection of the HPV 16/18 vaccines provides also long-lasting protection against non-vaccine HPV types such as 45,31 and 33, present in relevant percentages in the population. ${ }^{30} 31$

The strengths of our study is that all samples derived from the only Pathology laboratory in Curaçao where all cases were handled according to one protocol. Three pathologists did histological review and HPV typing was carried out following a single standardised protocol in DDL, which has ample experience with HPV genotyping in very large epidemiological studies on genotyping. ${ }^{20}$

A limitation of the study is the limited number of CIN lesions studied because only available blocks of good quality and containing enough tissue were included. Consequently, this should be taken into account when interpreting the HPV prevalence data. However, this is the largest series of CIN from the Caribbean area in which HPV prevalence was assessed.

In conclusion, our study shows that compared with the world population the prevalence of HPV types 16 and 18 in cervical cancer is lower and consequently the contribution of the HPV genotypes $31,45,51,52$ and 58 is higher. When considering prophylactic HPV vaccination on Curaçao, these HPV genotypes' prevalence data in cervical (pre)cancer should be taken into account.

\section{Handling editor Jackie A Cassell}

Acknowledgements The authors would like to thank C.M.D. Coronel, pathologist, Ing. E. Benita, Cytotechnologist; laboratory and secretary personnel from Analytic Diagnostic Centre department of Pathology. The authors would also like to thank D.T Geraets, J. Roer, H. van den Munckhof, L. van den Berg, and the technical and laboratory personnel from DDL Diagnostic Laboratory and Dr J. Berkhof from VU University Medical Centre, Department of Epidemiology and Biostatistics for their expert help. Also, the authors would like to extend their gratitude to Dr. Gomes Bravio for providing information about cervical cancer screening and $\mathrm{S}$. Elhage, M. Ruijs and J. Boujon for their attribution on information about local vaccine availability.

Contributors Design of the study: Hooi, Witte, Kenter,Meijer, Quint. Data collection: Hooi, Pinedo. HPV detection and genotyping: de Koning, Quint, Hooi. Statistics: Hooi, Witte, Meijer, de Koning, Quint. Writing: Hooi, Meijer, Kenter, Witte, Quint. All authors critically commented on all versions of the manuscript.

Funding This work was financially supported by Fundashon Prevenshon grant number 1 .

Competing interests Desiree J. Hooi, no COI Birgit Lissenberg-Witte: no COI Maurits de Koning: no COI Herbert Micheal Pinedo: no COI Gemma Kenter: no 
COI Chris J.L.M. Meijer has received speakers fee from GSK, Qiagen, SPMSD/ Merck, Roche, Menarini and Seegene, served occasionally on the scientific advisory board (expert meeting) of GSK, Qiagen, SPMSD/Merck., Roche and Genticel and by occasion as consultant for Qiagen and Genticel. He is minority stock holder Self-Screen b.v. , a spin off company of VUMC. Until April 2016 he was minority stock holder of Diassay b.v. Until 2014 he had a small number of certificates of shares in Delphi Biosciences, which went into receivership in 2014 Wim Quint has obtained projects from GSK and Qiagen and is stockholder of DDL Diagnostic Laboratory.

Patient consent Detail has been removed from this case description/these case descriptions to ensure anonymity. The editors and reviewers have seen the detailed information available and are satisfied that the information backs up the case the authors are making.

Ethics approval The institutional review board of Fundashon Prevenshon, Curaçao, approved this study.

Provenance and peer review Not commissioned; externally peer reviewed.

Open Access This is an Open Access article distributed in accordance with the terms of the Creative Commons Attribution (CC BY 4.0) license, which permits others to distribute, remix, adapt and build upon this work, for commercial use, provided the original work is properly cited. See: http://creativecommons.org/licenses/by/4.0/

(c) Article author(s) (or their employer(s) unless otherwise stated in the text of the article) 2018. All rights reserved. No commercial use is permitted unless otherwise expressly granted.

\section{REFERENCES}

1 Central Bureau of Statistics Curaçao. Statistical yearbook. Willemstad,Curaçao: Central Bureau of Statistics Curaçao Fort Amsterdam z/n Curaçao, 2015. http://www.cbs.cw/ website/publications_231/rubriek/statistical-yearbook_62.html

2 Murillo R, Almonte M, Pereira A, et al. Cervical Cancer Screening Programs in Latin America and the Caribbean. Vaccine 2008;26:L37-L48.

3 Consedine NS, Tuck NL, Ragin CR, et al. Beyond the black box: a systematic review of breast, prostate, colorectal, and cervical screening among native and immigrant African-descent Caribbean populations. J Immigr Minor Health 2015; 17:905-24.

4 Ferlay J, Soerjomataram I, Dikshit R, et al. Cancer incidence and mortality worldwide: sources, methods and major patterns in GLOBOCAN 2012. Int J Cancer 2015;136:E3 59-E386.

5 Bosch FX, Lorincz A, Muñoz N, et al. The causal relation between human papillomavirus and cervical cancer. BMJ 2002.

6 zur Hausen H. Papillomaviruses in the causation of human cancers - a brief historical account. Virology 2009;384:260-5.

7 Walboomers JM, Jacobs MV, Manos MM, et al. Human papillomavirus is a necessary cause of invasive cervical cancer worldwide. J Pathol 1999;189:12-19.

8 Banydeen R, Rose AM, Martin D, et al. Advancing Cancer Control Through Research and Cancer Registry Collaborations in the Caribbean. Cancer Control 2015;22:520-30.

9 Lewis Merle J. A Situational analysis of Cervical Cancer Latin America and The Caribbean. Washington DC, 2004.

10 Martin D. Situational Analysis of Cervical Cancer Prevention and Contro/ Situational Analysis of Cervical Cancer, 2013.

11 Garland SM, Kjaer SK, Muñoz N, et al. Impact and Effectiveness of the Quadrivalent Human Papillomavirus Vaccine: A Systematic Review of 10 Years of Real-world Experience. Clin Infect Dis 2016;63:519-27.
12 Bosch FX, Tsu V, Vorsters A, et al. Reframing cervical cancer prevention. Expanding the field towards prevention of human papillomavirus infections and related diseases. Vaccine 2012;30:F1-F11.

13 Kleter B, van Doorn LJ, ter Schegget J, et al. Novel short-fragment PCR assay for highly sensitive broad-spectrum detection of anogenital human papillomaviruses. Am J Pathol 1998:153:1731-9.

14 Geraets DT, Grünberg AW, van der Helm JJ, et al. Cross-sectional study of genital carcinogenic HPV infections in Paramaribo, Suriname: prevalence and determinants in an ethnically diverse population of women in a pre-vaccination era. Sex Transm Infect 2014;90:627-33.

15 Kleter B, van Doorn LJ, Schrauwen L, et al. Development and clinical evaluation of a highly sensitive PCR-reverse hybridization line probe assay for detection and identification of anogenital human papillomavirus. J Clin Microbiol 1999:37:2508-17.

16 Luo W, Yang H, Rathbun K, et al. Detection of Human Immunodeficiency Virus Type 1 DNA in Dried Blood Spots by a Duplex Real-Time PCR Assay. J Clin Microbiol 2005;43:1851-7

17 Quint W, Jenkins D, Molijn A, et al. One virus, one lesion--individual components of CIN lesions contain a specific HPV type. J Pathol 2012;227:62-71.

18 Wentzensen N, Schiffman M, Dunn ST, et al. Multiple HPV genotype infections in cervical cancer progression in the Study to Understand Cervical Cancer Early Endpoints and Determinants (SUCCEED). NIH Public Access 2010;125:2151-8.

19 ICO HPV Information Centre. Human Papillomavirus and Related Diseases Report: HPV Information Centre. www.hpvcentre.net 15 December 2016.

20 de Sanjose S, Quint WG, Alemany L, et al. Human papillomavirus genotype attribution in invasive cervical cancer: a retrospective cross-sectional worldwide study. Lancet Oncol 2010;11:1048-56.

21 Guan P, Howell-Jones R, Li N, et al. Human papillomavirus types in 115,789 HPVpositive women: A meta-analysis from cervical infection to cancer. Int J Cancer 2012.

22 Hesselink AT, Berkhof J, van der Salm ML, et al. Clinical validation of the HPV-risk assay, a novel real-time PCR assay for detection of high-risk human papillomavirus DNA by targeting the E7 region. J Clin Microbiol 2014;52:890-6.

23 Wentzensen N, Vinokurova S, von Knebel Doeberitz M. Systematic review of genomic integration sites of human papillomavirus genomes in epithelial dysplasia and invasive cancer of the female lower genital tract. Cancer Res 2004;64:3878-84.

$24 \mathrm{Hu} \mathrm{Z}$, Zhu D, Wang W, et al. Genome-wide profiling of HPV integration in cervical cancer identifies clustered genomic hot spots and a potential microhomologymediated integration mechanism. Nat Genet 2015;47:158-63.

25 Santos GD, Michel M, Ekindi N, et al. Human Papillomavirus (HPV) genotype distribution in invasive cervical cancers in Martinique (French West Indies) abstract. Eurogin 2015;16:1P235

26 Hosein F, Mohammed W, Zubach V, et al. Human papillomavirus genotypes in invasive cervical squamous cell carcinoma in Trinidad. Rev Panam Salud Publica 2013;33:267-70.

27 Rattray C, Strickler HD, Escoffery C, et al. Type-specific prevalence of human papillomavirus DNA among Jamaican colposcopy patients. J Infect Dis 1996:173:718-21.

28 Lewis-Bell K, Luciani S, Unger ER, et al. Genital human papillomaviruses among women of reproductive age in Jamaica. Rev Panam Salud Publica 2013;33:159-65.

29 Cordel N, Ragin C, Trival M, et al. High-risk human papillomavirus cervical infections among healthy women in Guadeloupe. Int J Infect Dis 2015;41:13-16.

30 Harper DM, Franco EL, Wheeler CM, et al. Sustained efficacy up to 4.5 years of a bivalent $L 1$ virus-like particle vaccine against human papillomavirus types 16 and 18 : follow-up from a randomised control trial. The Lancet 2006;367:1247-55.

31 Malagón T, Drolet M, Boily MC, et al. Cross-protective efficacy of two human papillomavirus vaccines: a systematic review and meta-analysis. Lancet Infect Dis 2012;12:781-9. 